\title{
Formale und semantische Unschärfen in vielgliedrigen Verbalkomplexen \\ Der Ersatzinfinitiv und anderes
}

Per B/erentzen

Institut für Sprache, Literatur und Kultur, Abteilung für Deutsch, Universität Aarhus, Dänemark

I forskellige germanske sprog opviser mangeleddede verbalkomplekser, dvs. verbalkomplekser med mere end to verbalformer, undertiden formale og deraf afledte semantiske afvigelser $i$ forhold til normalkonstruktionerne, som de foreligger $\mathrm{i}$ de blot toleddede verbalkomplekser. De formale afvigelser lader sig beskrive ved hjælp af tre afvigelsesprincipper, der indfanger de påvirkninger, som verbalformerne i de mangeleddede komplekser udøver på hinanden. De semantiske følgevirkninger vedrører først og fremmest temporalsemantiske forhold. En fra tysk velkendt afvigelsestype er den såkaldte erstatningsinfinitiv, der også - om end sjældnere - forekommer i bl.a. dansk. Den har i tysk en kompliceret udviklingshistorie og tillige i bestemte tilfælde en ordstillingsmæssig effekt, der er begrundet i behovet for en sikker aflæsning af sætningers temporalitet.

\section{EINLEITUNG}

Als vielgliedrige Verbalkomplexe bezeichnen wir Verbalkomplexe mit mehr als zwei Verbformen. Verbalkomplexe mit nur zwei Verbformen bezeichnen wir im Gegensatz hierzu als einfache Verbalkomplexe. In einigen germanischen Sprachen lassen sich in vielgliedrigen Verbalkomplexen bisweilen formale und damit zusammenhängende semantische Unschärfen beobachten.

Im Folgenden werden zuerst das Grundprinzip und die drei Abweichungsprinzipien, die für die formale Strukturierung vielgliedriger Verbalkomplexe gültig sind (Abschnitt 2.), sowie Beispiele abweichender Strukturen aus dem Deutschen (2.1.), dem Dänischen (2.2.) und dem Afrikaans (2.3.) beschrieben. Anschließend wird auf semantische, vor allem die Temporalität betreffende Unschärfen eingegangen, die aus den formalen Abweichungen resultieren (Abschnitt 3.). Zum Schluss wird die Entstehung des deutschen Ersatzinfinitivs samt den von ihm herrührenden topologischen Besonderheiten etwas ausführlicher diskutiert (Abschnitt 4.). 


\section{StRUKTURIERUNGSPRINZIPIEN}

Im Hinblick auf die Beschreibung der formalen Strukturierung der Verbalkomplexe wird jede Verbform zur Kennzeichnung ihres hierarchischen Ranges innerhalb des Verbalkomplexes mit einer Indexziffer versehen. Ziffer 1 kommt der ranghöchsten Verbform zu. Zwischen zwei hierarchisch benachbarten Verbformen eines Verbalkomplexes liegt nach Bech (1955: 15f u. 25) prinzipiell ein Rektionsverhältnis vor, das darin besteht, dass die ranghöhere Verbform die morphologische Gestalt der rangniedrigeren Verbform bestimmt.

Für die formale Strukturierung vielgliedriger Verbalkomplexe lassen sich ein Grundprinzip und drei Abweichungsprinzipien formulieren. Die formalen (und semantischen) Unschärfen entstehen, wenn bei der Bildung eines vielgliedrigen Verbalkomplexes vom Grundprinzip abgewichen wird:

Grundprinzip: Jede regierte Verbform eines vielgliedrigen Verbalkomplexes erhält die Form, welche die ihr übergeordnete Verbform innerhalb eines einfachen Verbalkomplexes regieren würde. Im vielgliedrigen Verbalkomplex in (1) regiert $k a n n^{1}$ den $\varnothing$-Infinitiv haben ${ }^{2}$ und dieser regiert das Partizip Perfekt geschlafen ${ }^{3}$. Diese Rektionsverhältnisse sind dieselben wie in den beiden einfachen Verbalkomplexen in (1a) und (1b):

1) er kann ${ }^{1}$ nicht geschlafen ${ }^{3}$ haben ${ }^{2}$

1a) er kann ${ }^{1}$ es haben ${ }^{2}$

1b) er hat geschlafen $^{2}$

Abweichungsprinzipien: In vielgliedrigen Verbalkomplexen kann oder muss unter Umständen vom Grundprinzip abgewichen werden. Die Abweichung besteht darin, dass eine oder mehrere regierte Verbformen eine andere als die nach dem Grundprinzip zu erwartende morphologische Form erhalten. Es lassen sich drei Abweichungsprinzipien unterscheiden:

Abweichungsprinzip A:

Eine regierte Verbform kopiert die morphologische Form der ihr untergeordneten Verbform.

Abweichungsprinzip B:

Eine regierte Verbform kopiert die morphologische Form der ihr übergeordneten Verbform.

Abweichungsprinzip C:

Es werden zwei hierarchisch benachbarte regierte Verbformen zugleich betroffen. Die ranghöhere Verbform tritt in einer Reduktionsform auf und die rangniedrigere Verbform erscheint in der morphologischen Form, in der die ranghöhere Verbform nach dem Grundprinzip zu erscheinen 
hätte. Die beiden Verbformen werden sozusagen als eine Einheit flektiert.

Das Auftreten der Abweichungsprinzipien wird im Folgenden an Beispielen des Deutschen, des Dänischen und des Afrikaans demonstriert.

\subsection{Abweichende Strukturen im Deutschen}

In einigen Fällen realisieren gewisse Verben des Deutschen bei der Bildung des Perfekts und des Plusquamperfekts statt des zu erwartenden Partizips Perfekt den Infinitiv. Voraussetzung für das Auftreten dieses so genannten „Ersatzinfinitivs“ ist, dass von ihm ein weiterer Infinitiv abhängt. Dieser Fall ist eine Abweichung nach Prinzip A. In (2) kopiert die regierte Verbform können ${ }^{2}$ die morphologische Form der ihr untergeordneten Verbform kommen ${ }^{3}$ und erscheint somit als Ø-Infinitiv (Ersatzinfinitiv). Nach dem Grundprinzip hätte das Modalverb als Partizip Perfekt erscheinen sollen, wie aus dem einfachen Verbalkomplex in (2a) hervorgeht:

2) er hat ${ }^{1}$ nicht kommen können $^{2}$

2a) er hat ${ }^{1}$ es nicht gekonnt ${ }^{2}$

Auf die Entstehung des deutschen Ersatzinfinitivs und die mit ihm verbundenen topologischen Besonderheiten wird weiter unten (Abschnitt 4.) näher eingegangen.

Im Deutschen kommen auch Abweichungen nach Prinzip C vor. Das ist in (3) der Fall, wo die hierarchisch benachbarten Verbformen haben ${ }^{2}$ und zu können ${ }^{3}$ beide betroffen sind. Die rangniedrigere Verbform zu können ${ }^{3}$, die nach dem Grundprinzip (3a) als Partizip Perfekt hätte erscheinen sollen, erscheint in der morphologischen Form (zuInfinitiv), in der die ranghöhere Verbform haben ${ }^{2}$ nach dem Grundprinzip (3b) hätte erscheinen sollen. Die Verbform haben ${ }^{2}$ in (3) ist eine Reduktionsform (ein reduzierter $z u$-Infinitiv, also kein Ø-Infinitiv). Ein Vergleich mit (3c) zeigt außerdem, dass die Verbform zu können ${ }^{3}$ in (3) zugleich ein Ersatzinfinitiv ist, der in diesem besonderen Fall nicht als $\varnothing$-Infinitiv, sondern als $z u$-Infinitiv erscheint:

3) sie glaubt ${ }^{1}$ ihm damit haben ${ }^{2}$ helfen ${ }^{4}$ zu können ${ }^{3}$

3a) sie hat ${ }^{1}$ es gekonnt ${ }^{2}$ - *sie hat ${ }^{1}$ es zu können ${ }^{2}$

3b) sie glaubt ${ }^{1}$ es zu haben ${ }^{2}$ - *sie glaubt ${ }^{1}$ es haben ${ }^{2}$

3c) (sie glaubt,) dass sie ihm damit hat ${ }^{1}$ helfen $^{3}$ können $^{2}$

Wie (3) ist auch (4) zu beschreiben, wo die Reduktionsform haben ${ }^{2}$ zwar nicht von einem Verb, sondern von der Präposition ohne regiert ist, die wir deshalb mit der Indexziffer 1 versehen haben. Auch in diesem Fall hätte haben ${ }^{2}$ nach dem Grundprinzip (4a) als zuInfinitiv erscheinen sollen. Außerdem ist zu können ${ }^{3}$ in (4) ein Ersatzinfinitiv, der nicht als $\varnothing$-Infinitiv, sondern als $z \boldsymbol{u}$-Infinitiv erscheint:

4) ohne $e^{1}$ hm damit haben ${ }^{2}$ helfen ${ }^{4}$ zu können ${ }^{3}$

4a) ohner es zu haben ${ }^{2}$ *ohne es haben ${ }^{2}$ 
Eine Abweichung nach Prinzip C kommt im Deutschen auch beim Handlungspassiv in den perfektischen Tempora vor, wie es in (5) der Fall ist, wo die hierarchisch benachbarten Verbformen worden ${ }^{2}$ und gelobt ${ }^{3}$ beide betroffen sind. Die ranghöhere Verbform worden ${ }^{2}$ hat ihr Flexiv ge- eingebüßt und tritt somit in einer Reduktionsform auf, während die rangniedrigere Verbform gelobt ${ }^{3}$ in der morphologischen Form des Partizips Perfekt erscheint, in der sie zwar schon nach dem Grundprinzip (5a) zu erscheinen hätte, in der aber auch die ranghöhere Verbform nach dem Grundprinzip (5b) hätte erscheinen sollen:

5) sie sind ${ }^{1}$ häufig gelobt ${ }^{3}$ worden $^{2}$

5a) sie wurden ${ }^{1}$ häufig gelobt ${ }^{2}$

5b) sie sind ${ }^{1}$ krank geworden ${ }^{2}$ - *sie sind ${ }^{1}$ krank worden ${ }^{2}$

\subsection{Abweichende Strukturen im Dänischen}

Das Dänische weist in vielgliedrigen Verbalkomplexen, die semantisch deutschen Sätzen wie (2) mit Ersatzinfinitiv entsprechen, verschiedene Variationsmöglichkeiten auf. Das Dänische kann in solchen Fällen entweder nach dem Grundprinzip oder nach allen drei Abweichungsprinzipien A, B und C konstruieren. Die Konstruktion in (6), die dem Grundprinzip folgt, ist die standarddänische Normalkonstruktion. Die hiervon abweichenden Konstruktionen in (7) bis (10) sind (noch) nicht als standarddänisch zu betrachten und kommen vor allem in der gesprochenen Sprache vor.

Dass die Konstruktion in (6) dem Grundprinzip folgt, zeigt der Vergleich mit den einfachen Verbalkomplexen in (6a) und (6b). Die Verbform kunnet ${ }^{2}$ ist ein von har ${ }^{1}$ regiertes Partizip Perfekt und die Verbform komme ${ }^{3}$ ist ein von kunnet $^{2}$ regierter $\varnothing$ Infinitiv:

6) han har ${ }^{1}$ ikke kunnet ${ }^{2}$ komme $^{3}$ 'er hat nicht gekonnt kommen' (= er hat nicht kommen können)

6a) han har ikke kunnet ${ }^{2}$ det 'er hat nicht gekonnt es' (= er hat es nicht gekonnt)

6b) han kunne ikke komme ${ }^{2}$ 'er konnte nicht kommen'

Die Konstruktion in (7) ist eine Abweichung nach Prinzip A und entspricht der deutschen Konstruktion mit Ersatzinfinitiv. Die regierte Verbform skulle ${ }^{2}$ kopiert die morphologische Form der ihr untergeordneten Verbform betale ${ }^{3}$ und erscheint somit als $\varnothing$-Infinitiv, hätte aber nach dem Grundprinzip als Partizip Perfekt erscheinen sollen, wie aus dem einfachen Verbalkomplex in (7a) hervorgeht:

7) vi har ${ }^{1}$ altid skulle betale $^{3}$ dyre portobeløb 'wir haben immer sollen bezahlen teure Portobeträge' (= wir haben immer teure Portobeträge bezahlen müssen) 
7a) vi har ${ }^{1}$ altid skullet ${ }^{2}$ det - *vi har ${ }^{1}$ altid skulle ${ }^{2}$ det

'wir haben immer gesollt es' (= wir haben es immer gesollt)

Die Konstruktion in (8) ist eine Abweichung nach Prinzip B. Die regierte Verbform havde ${ }^{2}$ kopiert die morphologische Form der ihr übergeordneten Verbform burde 1 und erscheint somit als finite Verbform, hätte aber nach dem Grundprinzip als Ø-Infinitiv erscheinen sollen, wie aus dem einfachen Verbalkomplex in (8a) hervorgeht. Auffällig ist, dass die Abweichung dazu führt, dass Satz (8) zwei Finita enthält:

8) bagefter burde jeg måske havde kunnet $^{3}$ forudse $^{4}$, at ...

'nachher sollte ich vielleicht hatte gekonnt voraussehen, dass ...'

(= nachher hätte ich vielleicht voraussehen können müssen, dass ...)

8a) jeg burde have ${ }^{2}$ det - *jeg burde ${ }^{1}$ havde ${ }^{2}$ det

'ich sollte haben es' (= ich sollte es haben)

Auch die Konstruktion in (9) ist eine Abweichung nach Prinzip B. Die regierte Verbform spurgt ${ }^{3}$ kopiert die morphologische Form der ihr übergeordneten Verbform villet $^{2}$ und erscheint somit als Partizip Perfekt, hätte aber nach dem Grundprinzip als Ø-Infinitiv erscheinen sollen, wie aus dem einfachen Verbalkomplex in (9a) hervorgeht:

9) det har ${ }^{1}$ jeg hele tiden villet ${ }^{2}$ spurgt $^{3}$ om

'da habe ich immer gewollt gefragt nach'

(= danach habe ich immer fragen wollen)

9a) det vil ${ }^{1}$ jeg spørge ${ }^{2}$ om - *det vil ${ }^{1}$ jeg spurgt ${ }^{2}$ om

'da will ich fragen nach'

Die Konstruktion in (10) ist eine Abweichung nach Prinzip C. Die hierarchisch benachbarten Verbformen $k u^{2}$ und sovet ${ }^{3}$ sind beide betroffen. Die rangniedrigere Verbform sovet ${ }^{3}$, die nach dem Grundprinzip (10a) als Ø-Infinitiv hätte erscheinen sollen, erscheint in der morphologischen Form (Partizip Perfekt), in der die ranghöhere Verbform $k u^{2}$ nach dem Grundprinzip (10b) hätte erscheinen sollen. Die Verbform $k u^{2}$ in (10) ist eine Reduktionsform (ein reduziertes Partizip Perfekt):

10) han har ${ }^{1}$ aldrig $k u^{2}$ sovet $^{3}$ om dagen

'er hat nie könn- geschlafen am Tage'

(= er hat nie am Tage schlafen können)

10a) han $\mathrm{kan}^{1}$ aldrig sove ${ }^{2}$ - *han $\mathrm{kan}^{1}$ aldrig sovet ${ }^{2}$

'er kann nie schlafen'

10b) han har ${ }^{1}$ aldrig kunnet ${ }^{2}$ det - *han har ${ }^{1}$ aldrig $k u^{2}$ det

'er hat nie gekonnt es' (= er hat es nie gekonnt) 


\subsection{Abweichende Strukturen im Afrikaans}

Im Afrikaans sind die Verhältnisse besonders kompliziert, weil die Verben - mit Ausnahme von hê 'haben' und wees 'sein' - nur zwei Flexionsformen unterscheiden. Die eine Flexionsform ist eine Stammform, die sich als Infinitiv und als finite Präsensform zugleich betrachten lässt. Die andere Flexionsform ist bei den Modalverben eine finite Imperfektform, bei den übrigen Verben ein Partizip Perfekt. Die beiden Formen der Modalverben sind nur zu einem gewissen Grad als Tempusformen zu betrachten, da sie häufig alternieren können, ohne einen temporalsemantischen Unterschied herbeizuführen. Das Verb hê besitzt neben diesem Infinitiv die finite Präsensform het und das Partizip Perfekt gehad (vgl. Donaldson 1993: 218f u. 239f).

Die Konstruktion in (11) lässt sich als eine Abweichung nach Prinzip A beschreiben und entspricht der deutschen Konstruktion mit Ersatzinfinitiv. Die regierte Verbform $o p h o u^{2}$ kopiert die morphologische Form der ihr untergeordneten Verbform reën ${ }^{3}$ und erscheint somit als Ø-Infinitiv, hätte aber nach dem Grundprinzip als Partizip Perfekt erscheinen sollen, wie aus dem einfachen Verbalkomplex in (11a) hervorgeht:

11) dit het ${ }^{1}$ ophou ${ }^{2}$ rë̈n ${ }^{3}$

,es hat aufhören regnen' (= es hat zu regnen aufgehört)

11a) dit het ${ }^{1}$ opgehou ${ }^{2}$ * dit het ${ }^{1}$ ophou $^{2}$

'es hat aufgehört'

Die Konstruktionen in (12), (13), (14) und (15) zeigen Abweichungen nach Prinzip B. In (12) kopiert die regierte Verbform $h \mathrm{t}^{2}$ die morphologische Form der ihr übergeordneten Verbform sal ${ }^{1}$ und erscheint eindeutig als finite Verbform, hätte aber nach dem Grundprinzip als $\varnothing$-Infinitiv erscheinen sollen, wie aus dem einfachen Verbalkomplex in (12a) hervorgeht. Auffällig ist, dass die Abweichung dazu führt, dass Satz (12) zwei Finita enthält, die beide im Präsens stehen. In (13) kopiert die regierte Verbform $k^{2}{ }^{2}$ die morphologische Form der ihr übergeordneten Verbform sou ${ }^{1}$ und der Satz weist somit zwei finite Verbformen auf, die beide im Imperfekt stehen. In (14) kopiert die regierte Verbform $h^{2} t^{2}$ die morphologische Form der ihr übergeordneten Verbform sou und erscheint somit als finite Verbform, hätte aber nach dem Grundprinzip als Ø-Infinitiv erscheinen sollen, wie aus dem einfachen Verbalkomplex in (14a) hervorgeht. Auch in diesem Fall führt die Abweichung also zu einem Satz mit zwei finiten Verbformen, von denen jedoch die übergeordnete $\left(s o u^{1}\right)$ im Imperfekt, die untergeordnete $\left(h t^{2}\right)$ im Präsens steht. Auch Satz (15) zeigt eine Abweichung nach Prinzip B mit zwei finiten Verbformen, von denen jedoch die übergeordnete $\left(h e t^{1}\right)$ im Präsens, die regierte $\left(k o n^{2}\right)$ im Imperfekt steht:

12) hy sal ${ }^{1}$ dit voor dié tyd gedoen ${ }^{3}$ het $^{2}$

'er wird es bis dahin getan hat' (= er wird es bis dahin getan haben)

12a) hy sal dit én dag hêt - *hy sal dit én dag het ${ }^{2}$

'er wird es eines Tages haben' 
13) ek sou dit kon ${ }^{2}$ doen ${ }^{3}$

'ich sollte es konnte tun' (= ich würde es tun können)

14) hy sou dit gedoen ${ }^{3}$ het $^{2}$

'er sollte es getan hat' (= er würde es getan haben)

14a) hy sou dit hê $\hat{e}^{2}{ }^{*}$ hy sou' dit het $^{2}$

'er sollte es haben' (= er würde es haben)

15) ek het dit altyd $\mathrm{kon}^{2}$ doen ${ }^{3}$

'ich habe es immer konnte tun' (= ich habe es immer tun können)

Die Konstruktionen in (16) und (17) lassen sich auf zweierlei Weise beschreiben, da die regierte Verbform $k a n^{2}$ sich sowohl als Infinitiv wie auch als finite Präsensform interpretieren lässt. Wird $\mathrm{kan}^{2}$ als Infinitiv interpretiert, weisen (16) und (17) eine Abweichung nach Prinzip A auf und sind wie (11) zu beschreiben. Wird $\mathrm{kan}^{2}$ als finite Präsensform interpretiert, weisen (16) und (17) eine Abweichung nach Prinzip B auf. Dann ist Satz (16) wie (12) zu beschreiben, da er zwei finite Verbformen (beide im Präsens) enthält, während Satz (17) wie (14) zu beschreiben ist, da er zwei finite Verbformen (im Imperfekt bzw. Präsens) enthält:

16) ek het dit altyd $\mathrm{kan}^{2}$ doen ${ }^{3}$

'ich habe es immer können/kann tun'

(= ich habe es immer tun können)

17) ek sou dit kan ${ }^{2}$ doen $^{3}$

'ich sollte es können/kann tun' (= ich würde es tun können)

\section{Semantische UnSChärfeN}

Aus den formalen Abweichungen innerhalb der Verbalkomplexe resultieren semantische Unschärfen, die vor allem die Temporalität betreffen. Besonders auffällig sind die semantischen Unschärfen in Verbalkomplexen, in denen mithilfe des Auxiliars haben (bzw. dänisch have und afrikaans hê) die Vergangenheit ausgedrückt werden soll.

Während im einfachen (zweigliedrigen) Verbalkomplex die Vergangenheit durch die Kombination Auxiliar haben + Partizip Perfekt des Hauptverbs (er hat ${ }^{1}$ geschlafen $^{2}$ ) ausgedrückt wird, kann sie in strukturell abweichenden vielgliedrigen Verbalkomplexen entweder durch eine defektive oder eine pleonastische Temporalkonstruktion oder auf andere Weise ausgedrückt sein. Wir wollen das anhand des oben behandelten Beispielmaterials veranschaulichen.

Eine defektive Temporalkonstruktion liegt in den folgenden Fällen vor, in denen die Vergangenheit allein durch das Auxiliar haben/have/hê ausgedrückt wird, da die vom Auxiliar abhängige Verbform nicht in der nach dem Grundprinzip zu erwartenden Form des Partizips Perfekt, sondern als Infinitiv erscheint: 
2) er hat ${ }^{1}$ nicht kommen ${ }^{3}$ können ${ }^{2}$

3) sie glaubt ${ }^{1}$ ihm damit haben ${ }^{2}$ helfen ${ }^{4}$ zu können ${ }^{3}$

7) vi har ${ }^{1}$ altid skulle betale $^{3}$ dyre portobeløb

'wir haben immer sollen bezahlen teure Portobeträge'

(= wir haben immer teure Portobeträge bezahlen müssen)

11) dit het ${ }^{1}$ ophou $^{2}$ reёn ${ }^{3}$

,es hat aufhören regnen' (= es hat zu regnen aufgehört)

16) ek het ${ }^{1}$ dit altyd $\mathrm{kan}^{2}$ doen $^{3}$

'ich habe es immer können/kann tun'

(= ich habe es immer tun können)

Eine pleonastische Temporalkonstruktion liegt in (8) und (9) vor. In (8) ist die Vergangenheit sowohl im präteritalen Finitum havde ${ }^{2}$, das nach dem Grundprinzip als Infinitiv zu erscheinen hätte, als auch im Partizip Perfekt kunnet $^{3}$ ausgedrückt. In (9) ist die Vergangenheit in der normalen Temporalkonstruktion har $^{1}$ villet $^{2}$ und zusätzlich im Partizip Perfekt spurgt ${ }^{3}$ ausgedrückt:

8) bagefter burde ${ }^{1}$ jeg måske havde kunnet $^{3}$ forudse $e^{4}$, at ...

'nachher sollte ich vielleicht hatte gekonnt voraussehen, dass ...'

(= nachher hätte ich vielleicht voraussehen können müssen, dass ...)

9) det har ${ }^{1}$ jeg hele tiden villet ${ }^{2}$ spurgt $^{3}$ om

'da habe ich immer gewollt gefragt nach'

(= danach habe ich immer fragen wollen)

Weitere semantische Unschärfen liegen in (10) sowie in den Zeitungsbelegen (18) und (19) vor. In (10) ist die Vergangenheit durch das Auxiliar har ${ }^{1}$ in Kombination mit dem Partizip Perfekt sovet ${ }^{3}$ ausgedrückt. Auffällig ist in diesem Fall, dass die vom Auxiliar unmittelbar abhängige Verbform $k u^{2}$ als Reduktionsform erscheint und sich nicht am temporalen Ausdruck beteiligt. In (18) und (19) ist die Vergangenheit durch das Auxiliar hätte $(n)^{1}$ ausgedrückt. Das Modalverb können ${ }^{2}$ ist ein Ersatzinfinitiv, der als solcher keinen Beitrag zum temporalen Ausdruck leistet. Als Kompensation hierfür wird die Vergangenheit zusätzlich in der perfektischen Konstruktion gewesen ${ }^{4} \operatorname{sein}^{3}$ ausgedrückt:

10) han har ${ }^{1}$ aldrig $k u^{2}$ sovet $^{3}$ om dagen

er hat nie könn- geschlafen am Tage

(= er hat nie am Tage schlafen können)

18) [...] mit Bachs Partita Nr. 1 in h-Moll, die Hohenthal zwar aufeinem modernen Instrument spielte, in der zarten Klanglichkeit, mit der er sich der enigmatischen Polyphonie stellte, hätten ${ }^{1}$ es aber auch Darmsaiten gewesen ${ }^{4}$ sein $^{3}$ können ${ }^{2}$, die er strich.

19) Und auch nach der Wende [...] blieben die Grenzen zu undurchlässig, als dass die Lage Zittaus für größere Industrieansiedelungen wie die in Leipzig oder Dresden hätte ${ }^{1}$ interessant gewesen ${ }^{4}$ sein $^{3}$ können $^{2}$. 


\section{Ersatzinfinitiv und Oberfeld im Deutschen}

Mit dem Auftreten des Ersatzinfinitivs ist im Deutschen eine topologische Besonderheit verknüpft. Die Abfolge mehrerer Verbformen im Schlussfeld des Satzes wird grundsätzlich durch den hierarchischen Rang der Verbformen bestimmt, so dass ein ranghöheres Verb (mit einer niedrigeren Indexziffer) rechts vom jeweils rangniedrigeren Verb (mit einer höheren Indexziffer) steht, aber von dieser Grundregelung wird in einigen Fällen obligatorisch oder fakultativ abgewichen. Bei jedem Ersatzinfinitiv ist das temporale Hilfsverb haben vorhanden und dem Ersatzinfinitiv hierarchisch übergeordnet. Falls das Hilfsverb nicht das Finitum eines Kern- oder Stirnsatzes ist, befindet es sich mit dem Ersatzinfinitiv und (mindestens) einem weiteren Infinitiv zusammen im Schlussfeld des Satzes und muss dann gegen die Grundregelung links von den ihm hierarchisch untergeordneten Verbformen stehen, wie aus (20) und (21) hervorgeht. In (22) und (23), wo neben dem Finitum wird zwei Infinitive, jedoch kein Ersatzinfinitiv, vorhanden sind, liegt eine fakultative Umstellung innerhalb des Schlussfeldes vor, da das Finitum wird rechts oder links von den ihm hierarchisch untergeordneten Verbformen stehen kann. Nach Bech (1955: 62f) lässt sich das Schlussfeld in ein Oberfeld und ein Unterfeld aufteilen. Das Oberfeld macht den linken Teil, das Unterfeld den rechten Teil des Schlussfeldes aus. In den folgenden Beispielen stehen die gesperrtgedruckten Verbformen im Oberfeld, die übrigen Verbformen des Schlussfeldes in dessen Unterfeld:

20) weil er nicht hat ${ }^{1}$ kommen $^{3}$ können $^{2}$

20a) *weil er nicht kommen ${ }^{3}$ können ${ }^{2}$ hat $^{1}$

21) er soll nicht haben ${ }^{2}$ kommen $^{4}$ können $^{3}$

21a) *er soll ${ }^{1}$ nicht kommen ${ }^{4}$ können ${ }^{3}$ haben $^{2}$

22) dass sie hier arbeiten ${ }^{3}$ können ${ }^{2}$ wird $^{1}$

23) dass sie hier wird ${ }^{1}$ arbeiten $^{3}$ können $^{2}$

Es scheint zwischen dem Vorkommen des Ersatzinfinitivs und der obligatorischen Oberfeldplatzierung des Hilfsverbs haben in Fällen wie (20) und (21) eine enge Korrelation zu bestehen, weshalb sich die Frage stellt, ob zwischen den beiden Phänomenen ein Kausalverhältnis besteht, also: Ist die Oberfeldbildung eine Folge des Ersatzinfinitivs oder umgekehrt?

Hierzu meinen Eisenberg/Smith/Teuber (2001), dass eine Erklärung der Umstellungen im Schlussfeld bei den obligatorischen Fällen mit haben + Ersatzinfinitiv wie in (20) und (21) zu suchen ist und dass die Verwendung des Ersatzinfinitivs die Oberfeldplatzierung des Hilfsverbs haben verursacht. Sie sagen:

"Wir müssen [...] nur zeigen, warum das Perfekt der Modalverben nicht mit dem Partizip2 gebildet werden kann und warum als Folge dessen ein Oberfeld gebildet wird. Die Unmöglichkeit des Partizip2 sollte semantische Gründe haben, die Wahl des Ersatzinfinitivs sollte sich als Wahl der infiniten Defaultform erweisen und die Oberfeldbildung 
sollte sich als syntaktische Konsequenz daraus ergeben." (Eisenberg/Smith/Teuber 2001: 251)

Und weiter:

"Als nächster Bestandteil der Lösungsskizze hat eine Beschreibung des Partizip2 im Perfekt zu erfolgen, die geeignet ist, die Vermeidung des Partizips bei den Modalverben zu erklären." (Eisenberg/Smith/Teuber 2001: 252)

Eisenberg/Smith/Teuber (2001: 256) sehen in der Wahl des Infinitivs statt des Partizips Perfekt bei den Modalverben einen Reflex temporal-semantischer Verhältnisse. Es geht Ihnen darum, ob ein Verb im Stande ist, einen Nachzustand auszudrücken, und zwar entweder an seinem Subjekt oder an seinem Objekt. Es lassen sich drei Typen unterscheiden:

Ty p (a ) : Verben, die einen Nachzustand ihres Subjekts bezeichnen können. Sie bilden das Perfekt mit dem Hilfsverb sein:

erkranken: Der Freund ist erkrankt - der erkrankte Freund

zufrieren: Der See ist zugefroren - der zugefrorene See

Ty p ( b ) : Verben, die einen Nachzustand ihres Objekts bezeichnen können. Sie bilden das Perfekt mit dem Hilfsverb haben:

schließen: Der Freund hat das Fenster geschlossen - das geschlossene Fenster

streichen: Der Freund hat die Wand frisch gestrichen - die frisch gestrichene Wand

Typ (c) : Verben, die keinen Nachzustand bezeichnen können. Sie bilden das Perfekt mit dem Hilfsverb haben:

schlafen: Der Freund hat lange geschlafen - *der lange geschlafene Freund weinen: Das Kind hat eben geweint - *das eben geweinte Kind

Nach Eisenberg/Smith/Teuber (2001: 255f) entsprechen die Modalverben dem Typ (c), da sie keinen Nachzustand bezeichnen können. Deshalb sei bei ihnen die Selektion des Partizips Perfekt semantisch blockiert und das Perfekt könne deshalb nicht mit dem Partizip gebildet werden. An seiner Stelle trete dann die unmarkierte infinite Form, der $\varnothing$-Infinitiv.

Die Theorie von Eisenberg/Smith/Teuber weist jedoch einige Schwächen auf:

(i) Die Verben von Typ (c), die temporal-semantisch den Modalverben ähneln, bilden ja das Perfekt mit dem Partizip Perfekt und nicht mit dem Infinitiv:

$\begin{array}{ll}\text { schlafen: } & \begin{array}{l}\text { Der Freund hat lange geschlafen } \\ \text { *Der Freund hat lange schlafen }\end{array} \\ \text { weinen: } & \begin{array}{l}\text { Das Kind hat eben geweint } \\ \text { *Das Kind hat eben weinen }\end{array}\end{array}$


(ii) Die Modalverben bilden nur dann das Perfekt mit dem Partizip Perfekt, wenn sie als Hilfsverben benutzt werden, sonst bilden sie das Perfekt wie die anderen Verben mit dem Partizip Perfekt:

\section{können: $\quad$ Er hat nicht kommen können \\ ${ }^{*}$ Er hat nicht kommen gekonnt \\ Er hat den Text auswendig gekonnt \\ ${ }^{*}$ Er hat den Text auswendig können}

(iii) Falls tatsächlich die von Eisenberg/Smith/Teuber formulierte temporal-semantische Argumentation gültig sein sollte, wäre zu erwarten, dass in verwandten Sprachen die gleichen Regularitäten zu finden wären. Der Ersatzinfinitiv begegnet zwar in einigen, aber nicht in allen germanischen Sprachen.

Die Verwendung des Ersatzinfinitivs bei den Modalverben scheint also nicht, oder nicht ausschließlich, in der Temporalsemantik dieser Verben begründet zu sein. Wie im Vorausgehenden gezeigt wurde, besteht aber in mehreren Sprachen bei Verbalkomplexen mit mehr als zwei Verbformen häufig eine semantische Unschärfe, die aus einer formalen Unschärfe resultiert. Auch gehört zur Beschreibung der Entstehung des Ersatzinfinitivs im Deutschen neben der Semantik ein zweigliedriger formalhistorischer Erklärungsteil:

(i) Im älteren Deutsch besaß das perfektivierende Präfix ge- eine freiere Distribution als heute. Das Präfix konnte nicht nur beim Partizip Perfekt, sondern auch beim Infinitiv und bei finiten Verbformen gesetzt werden. Dal (1971: 196f) weist darauf hin, dass im Mittelhochdeutschen der Infinitiv nach Modalverben und nach Verben wie lâzen, heizen, helfen, horen, sehen häufig präfigiert war und dass ge- somit in großem Ausmaß den Infinitiv wie das Partizip Perfekt kennzeichnete. Dies führte in einigen starken Verbalklassen zum formalen Synkretismus zwischen Infinitiv und Partizip Perfekt, z.B. in Fällen wie gesehen, gevarn, geheizen und geloufen, so dass die Infinita in Fügungen wie ich hân gesehen (Partizip Perfekt) und ich mac gesehen (Infinitiv) gleichlautend sein konnten.

(ii) Wie Dal (1971: 199) anführt, scheint die Verbindung hoeren sagen, die in der epischen Sprache sehr häufig vorkam, den Ausgangspunkt für die Konstruktion mit Ersatzinfinitiv abgegeben zu haben. Ursprünglich wurde die Perfektbedeutung durch ein präfigiertes präteritales Finitum ausgedrückt, wie im Hildebrandslied ik gihôrta şat seggen 'ich habe das sagen hören'. Als dieses Präteritum durch das neue zusammengesetzte Perfekt ersetzt wurde, setzte man an Stelle des zu erwartenden Partizips Perfekt gehoret den Infinitiv 
horen, wie z.B. in von einem hân ich horen sagen (Biterolf). Dies geschah sicher in Analogie zu den häufig vorkommenden Fällen mit modalem Hilfsverb, wie z.B. muget ir nu wunder horen sagen (Nibelungenlied 1,4) und wurde außerdem durch die unter (i) erwähnten Fälle mit formaler Übereinstimmung von Infinitiv und Partizip Perfekt unterstützt.

Wie oben ausgeführt wurde, gehen Eisenberg/Smith/Teuber (2001) zu weit, wenn sie von den Modalverben behaupten, dass sie keinen Nachzustand bezeichnen können und dass das Perfekt deshalb mit dem Infinitiv statt des Partizips Perfekt gebildet werden muss. Dennoch ist bei den infiniten Verbformen in Verbalkomplexen eine gewisse semantische Unschärfe vorhanden, wie Dal bemerkt:

„In den beiden mhd. Fügungen ichmacgesehen :ich hângesehen gibt die Form gesehen nur den materiellen Bedeutungsinhalt der Handlung an, die Unterschiede in Zeit und Modalität werden in den Hilfsverba ausgedrückt." (Dal 1971: 198)

Zusammenfassend lässt sich sagen, dass der deutsche Ersatzinfinitiv also ursprünglich auf Grund gewisser formaler Synkretismen entstanden ist und dass er, wenn man andere germanische Sprachen mit einbezieht, keine einmalige Erscheinung ist, sondern sich in eine Reihe mit anderen Abweichungen vom formalen Grundprinzip stellt.

Dass das Auxiliar haben, wenn es mit zwei Infinitiven zusammen im Schlussfeld steht, obligatorisch ins Oberfeld rückt, erklärt sich daraus, dass der von haben abhängige Infinitiv ein Ersatzinfinitiv ist, der als solcher keinen Beitrag zum temporalen Ausdruck leistet. Da es aber wichtig zu sein scheint, dass man möglichst früh weiß, welches Tempus in einem Satz vorliegt, und da in Verbalkomplexen mit Ersatzinfinitiv allein das Auxiliar haben die Vergangenheit ausdrückt, wird haben ins Oberfeld gestellt, so dass man schon am Anfang des Verbalkomplexes und also vor dem Ende des Satzes dessen Tempus erahnen kann. Diese Auffassung vertreten auch Eisenberg/Smith/Teuber (2001: 257f). Deshalb wird (20), nicht aber (20a) realisiert:

20) weil er nicht hat ${ }^{1}$ kommen $^{3}$ können $^{2}$

20a) *weil er nicht kommen ${ }^{3}$ können ${ }^{2}$ hat $^{1}$

\section{LITERATUR}

Bech, Gunnar (1957), Studien über das deutsche verbum infinitum, Bd. I, København: Munksgaard (1983², Tübingen: Niemeyer).

Dal, Ingerid (1971), "Indifferenzformen im deutschen Verbalsystem", in: Dal, Ingerid (1971), Untersuchungen zur germanischen und deutschen Sprachgeschichte, Oslo, Bergen, Tromsö: Universitetsforlaget, pp. 194-221.

Donaldson, Bruce C. (1993), A Grammar of Afrikaans, Berlin, New York: Mouton de Gruyter. 
Bærentzen, Vielgliedrige Verbalkomplexe

Eisenberg, Peter, George Smith, Oliver Teuber (2001), "Ersatzinfinitiv und Oberfeld. Ein großes Rätsel der deutschen Syntax", Deutsche Sprache, 29 (2001), pp. 242-260. 